\section{Oral hygiene risk factor}

Sir, I would like to inform readers about the potential connection between high bacterial load in the mouth and complications associated with COVID-19 infection.

Oral hygiene should be improved during a COVID-19 infection in order to reduce the bacterial load in the mouth and the risk of a bacterial superinfection. We recommend that poor oral hygiene be considered a risk to COVID-19 complications, particularly in patients predisposed to altered biofilms due to diabetes, hypertension or cardiovascular disease. Bacteria present in patients with severe COVID-19 are associated with the oral cavity, and improved oral hygiene may reduce the risk of complications. Whilst COVID-19 has a viral origin, it is suspected that in severe forms of the infection, bacteria plays a part, increasing the chance of complications such as pneumonia, acute respiratory distress syndrome, sepsis, septic shock and death. ${ }^{1}$

The development and severity of complications following a COVID-19 infection depend on numerous host and viral factors that will affect a patient's immune response. Whilst
$80 \%$ of patients with COVID-19 infections have mild symptoms, $20 \%$ progress to have a severe form of infection associated with higher levels of inflammatory markers (Interleukin $2,6,10$ ) and bacteria., ${ }^{2,3}$ They also exhibit a remarkably higher neutrophil count and lower lymphocyte count than in mild patients. ${ }^{4} \mathrm{~A}$ high neutrophil count is abnormal for a viral infection, but common for a bacterial infection, suggesting that in severe cases of COVID-19, bacterial superinfection is common.

The three main comorbidities associated with an increased risk of complications from COVID-19 are diabetes, hypertension and cardiovascular disease. ${ }^{5}$ These comorbidities are also associated with altered oral biofilms and periodontal disease. Periodontopathic bacteria are implicated in systemic inflammation, bacteraemia, and pneumonia. ${ }^{6}$ Bacteria present in the metagenome of patients severely infected with COVID-19 included high reads for Prevotella, Staphylococcus, and Fusobacterium, all usually commensal organisms of the mouth. ${ }^{7}$ Over $80 \%$ of patients in ICU exhibited an exceptionally high bacterial load, ${ }^{3}$ and treatment has been successful with a dual regime of an antiviral and an antibiotic. ${ }^{8}$ It is clear that bacterial superinfections are common in patients suffering from a severe case of COVID-19.

V. Sampson, London, UK

\section{References}

1. World Health Organization. Clinical management of severe acute respiratory infection when COVID-19 is suspected. Interim guidance, 13 March 2020. Available at: https:// www.who.int/publications-detail/clinical-management-ofsevere-acute-respiratory-infection-when-novel-coronavirus(ncov)-infection-is-suspected (accessed April 2020).

2. Gong J. Correlation Analysis Between Disease Severity and Inflammation-related Parameters in Patients with COVID-19 Pneumonia. Tongji Hospital, 2020.

3. Liu J. Neutrophil-to-Lymphocyte Ratio Predicts Severe Illness Patients with 2019 Novel Coronavirus. Beijing Ditan Hospital, 2020

4. Zheng M, Gao Y, Wang G et al. Functional exhaustion of antiviral lymphocytes in COVID-19 patients. Cell Moll Immunol 2020; DOI: 10.1038/s41423-020-0402-2.

5. Zhou F, Yu T, Du R et al. Clinical course and risk factors for mortality of adult inpatients with COVID-19 in Wuhan, China: A retrospective cohort study. Lancet 2020; 395: 1054-1062.

6. Nagaoka K. Prevotella intermedia induces severe bacteremic pneumococcal pneumonia in mice with upregulated platelet-activating factor receptor expression. American Society for Microbiology, 2014

7. Chakraborty S. Metagenome of SARS-Cov2 patients in Shenzhen with travel to Wuhan: OSF Preprints, 2020.

8. Gautret P, Lagier J-C, Parola P et al. Hydroxychloroquine and azithromycin as a treatment of COVID-19: Results of an Open-Label Non-randomized trial. Int J Antimicrob Agents 2020; DOI: 10.1016/j.ijantimicag.2020.105949. https://doi.org/10.1038/s41415-020-1545-3

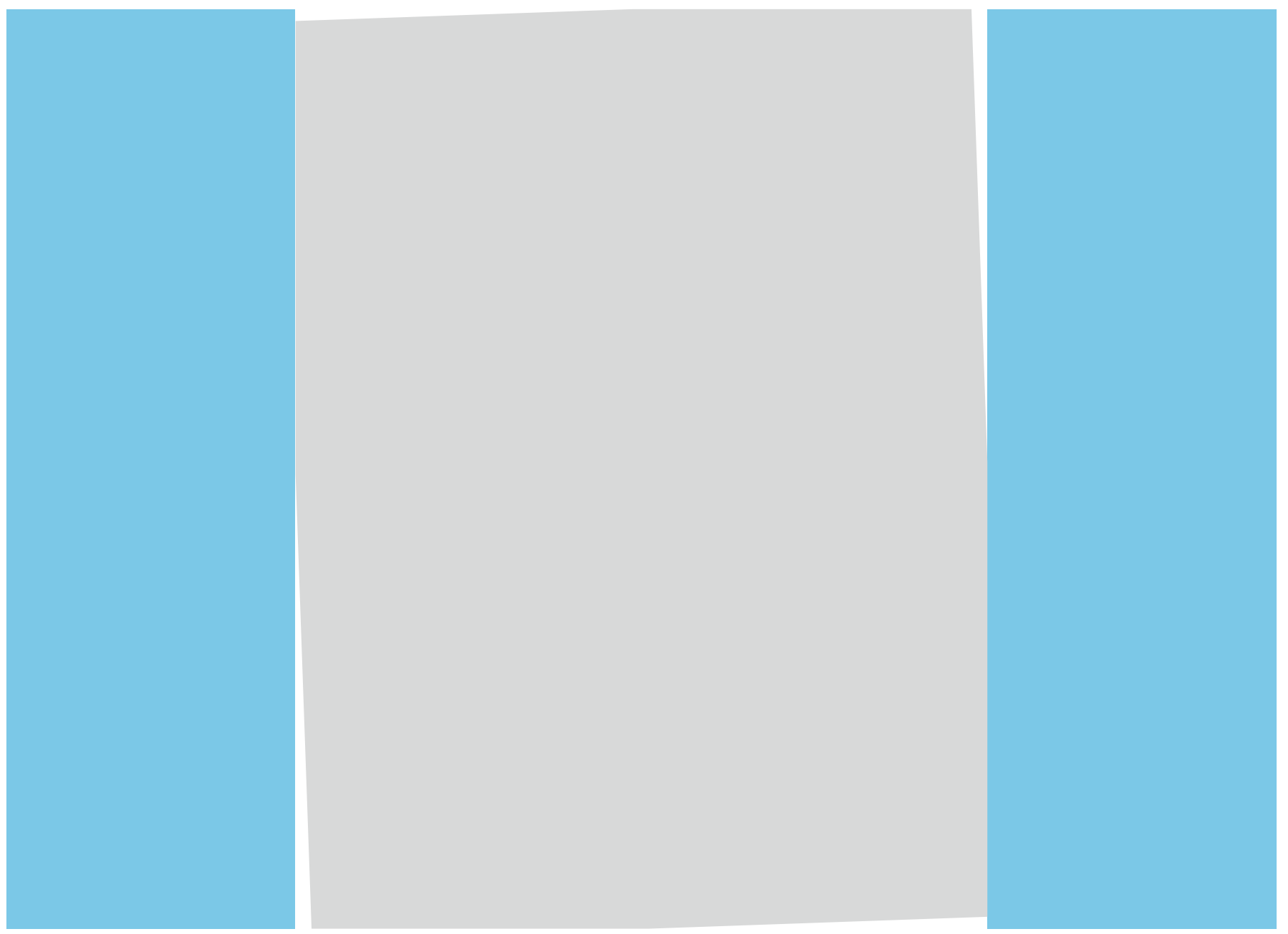

